УДК 517.9

MSC 41A60

\title{
COMPUTER PREDICTION OF INCREASING EFFICIENCY OF WORK OF RAPID MULTILAYER FILTERS FOR ADSORPTION PURIFICATION OF WATER
}

\author{
Andrij Bomba, Jurij Klimjuk
}

Department of Computer Science and Applied Mathematics, National University of Water and Environmental Engineering, Rivne, Ukraine, E-mail: abomba@ukr.net, klimyuk@ukr.net

\section{КОМП'ЮТЕРНЕ ПРОГНОЗУВАННЯ ПІДВИЩЕННЯ ЕФЕКТИВНОСТІ РОБОТИ ШВИДКИХ БАГАТОШАРОВИХ ФІЛЬТРІВ ДЛЯ АДСОРБЦІЙНОї ДООЧИСТКИ ВОДИ}

\author{
А. Я. БомБА, Ю. Є. КЛИмЮК
}

Кафедра комп'ютерних наук та прикладної математики, Національний університет водного господарства та природокористування, Рівне, Україна, E-mail: abomba@ukr.net, klimyuk@ukr.net

ABstract. A model of the adsorption purification of water from impurities in rapid multilayer cone-shaped filters with piecewisehomogeneous porous loads with a constant filtration rate taking into account the removal of part of the heat on the surfaces of division of layers is formulated. The proposed model provides an opportunity to conduct computer experiments to study the change in the concentrations of impurities in the filtration flow and adsorbed on the surface of the adsorbent load during physical and chemical adsorption, temperature, characteristics of piecewise-homogeneous porous loads along the filter height and based on their prediction of optimal adsorbent use and increasing the duration of filters.

KEYwORDS: mathematical model, water purification, adsorption, impurity, rapid multilayer filter.

АнотАція. Запропоновано модель адсорбційної доочистки води від домішок у швидких багатошарових фільтрах конусоподібної форми 3 кусково-однорідними пористими завантаженнями при сталій швидкості фільтрування з відведенням частини тепла 3 поверхонь розділу шарів. Модель забезпечує проведення комп'ютерних експериментів з вивчення зміни концентрацій домішок у фільтраційній течії та адсорбованих на поверхні адсорбенту завантаження при фізичній та хімічній адсорбції, температури, характеристик кусково-однорідних пористих завантажень вздовж висоти фільтру та прогнозування оптимальних варіантів використання адсорбентів і збільшення тривалості роботи фільтрів. КлючовІ СловА: математична модель, доочистка води, домішка, адсорбція, швидкий багатошаровий фільтр. 


\section{ВСТУП}

Швидкі фільтри для адсорбційної доочистки води від різних розчинених домішок застосовують зазвичай на заключній стадії водопідготовки, коли з неї відстоюванням, фільтрацією, коагуляцією вже видалена основна частина домішок [1-3]. Як адсорбенти використовують природні (бентоніт, монтморилоніт, торф), штучні (активоване вугілля, штучні цеоліти, полісорби) та синтетичні матеріали (наноструктуровані вуглецеві сорбенти) [4]. Підтримка постійної заданої швидкості фільтрування досягається автоматичним регулюванням за рахунок збільшення відкриття засувки на трубопроводі фільтрату в міру збільшення опору завантаження фільтра через накопичення в ньому частинок домішок. Імпульсом для збільшення відкриття засувки на трубопроводі фільтрату служить зміна рівня води на фільтрі (контролюється поплавковим пристроєм) або витрати води в трубопроводі фільтрату (контролюється за допомогою дроселюючого пристрою і дифманометра). Коли засувка відкрита повністю, фільтр вимикається 3 роботи для промивання [5]. Постійно зростаючі потреби у господарстві в очищеній воді та зростання вартості адсорбентів вимагають проведення досліджень їх більш оптимального використання і збільшення тривалості роботи фільтрів за рахунок вибору їх форми та висоти шарів, а також відведення частини тепла під час адсорбційної доочистки води.

Швидкість процесу адсорбції залежить від концентрації, природи і структури домішок, швидкості фільтрування, температури, виду і властивостей адсорбенту $[6,7]$. Залежно від характеру зв'язку адсорбент-адсорбат, тобто від вільної енергії взаємодії між адсорбційним центром адсорбенту і тією частинкою домішки, яка вступає в контакт з поверхнею адсорбенту, розрізняють фізичну і хімічну адсорбцію. При фізичній адсорбції частинки домішки прилипають до поверхні адсорбенту і утримуються на ній під дією сил міжмолекулярної взаємодії (сил Ван-дер-Ваальса), тобто при певних умовах можливе вилучення частинок домішки з адсорбенту. В основі процесу хімічної адсорбції лежить хімічна взаємодія між адсорбентом і частинкою домішки, тобто фактично при хімічній адсорбції змінюється хімічний склад поверхні адсорбенту. Процес хімічної адсорбції, як правило, є незворотнім, оскільки сили взаємодії при цьому значно вищі, ніж при фізичній адсорбції. Дослідження адсорбційної очистки води в умовах фізичного моделювання призводить до значних фінансових затрат, тому доцільним $є$ використання математичного моделювання на основі теорії адсорбційних і гідромеханічних процесів [8].

Як показує аналіз літературних джерел, зокрема [8-15], суттєвий вклад у розробку теоретичних основ адсорбційної очистки рідин від домішок зробило багато як вітчизняних, так і зарубіжних вчених. У [16] запропоновано просторову математичну модель для прогнозування процесу адсорбційної доочистки води від домішок у швидких багатошарових фільтрах конусоподібної форми при додержанні сталої швидкості фільтрування. Вона є більш ефективною для проведення теоретичних досліджень, націлених на оптимізацію параметрів процесу фільтрування (тривалості роботи, форми, розмірів фільтра, висоти шарів тощо) за рахунок не лише урахування i 
визначення концентрацій домішок відповідно у фільтраційній течії і на поверхні адсорбенту завантаження, але й введення додаткових рівнянь для визначення зміни температури фільтраційної течії, коефіцієнтів фільтрації та активної пористості завантаження, врахування дифузії домішок у фільтраційній течії і на поверхні зерен завантаження, урахування зміни швидкості фільтраційної течії вздовж висоти фільтру. Тому актуальною задачею є узагальнення відповідної моделі для комп'ютерного прогнозування процесу адсорбційної доочистки води від домішок у швидких багатошарових фільтрах з відведенням частини тепла з поверхонь розділу шарів.

\section{1. ПОСТАНОВКА ЗАДАЧІ ТА Ї̈̈ РОЗВ'ЯЗАННЯ}

Змоделюємо процес адсорбційної доочистки води від домішок у швидкому багатошаровому фільтрі конусоподібної форми із кусково-однорідним пористим завантаженням - однозв'язній області $G_{\mathbf{z}}(\mathbf{z}=(x, y, z))$, обмеженій гладкими, ортогональними між собою по ребрах, двома еквіпотенціальними поверхнями $S_{*}, S^{*}$ і поверхнею течії $S^{* *}$ та розділеній деякими заданими $p-1$ еквіпотенціальними поверхнями $S_{* r}^{*}(r=\overline{1, p-1})$ на $p$ підобластей $G_{\mathbf{z}}^{r}(r=\overline{1, p})$ (рис. 1 a).

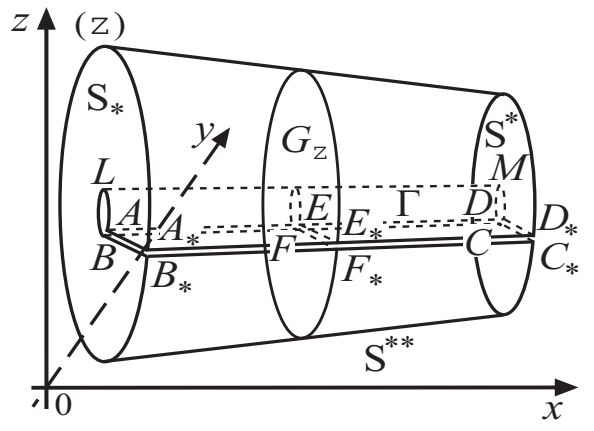

a)

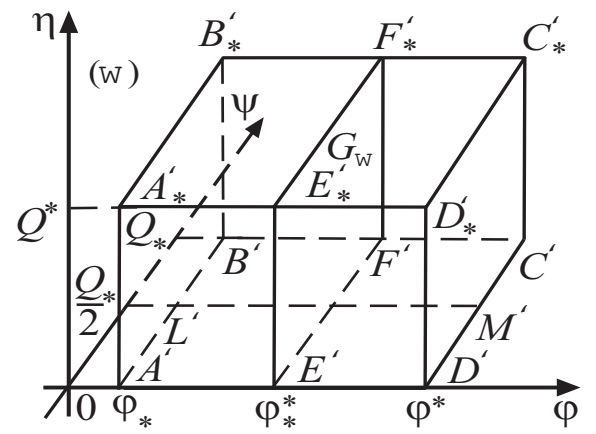

б)

Рис 1. Просторова двошарова область фільтрації (а) з умовним розрізом (конусоподібний фільтр) та область комплексного потенціалу (б)

Вважаємо, що конвективні складові тепло- і масоперенесення, швидкість масообміну при фізичній та хімічній адсорбції домішок переважають над вкладом дифузії й десорбції. Таким чином, для області $G=G_{\mathbf{z}} \times(0, \infty)$ відповідна просторова модельна задача з урахуванням зворотного впливу характеристик процесу (концентрацій домішок у фільтраційній течії та адсорбованих на поверхні адсорбенту завантаження за рахунок фізичної і хімічної адсорбції, температури) на характеристики завантаження (коефіцієнти фільтрації й активної пористості адсорбентів у кожному шарі, коефіцієнти, які характеризують швидкості масообміну при фізичній та хімічній адсорбції домішок і фізичній десорбції домішок) та відведення частини тепла з поверхонь розділу шарів складатиметься з систем рівнянь

$$
\vec{v}=\kappa_{0}^{0} \cdot \operatorname{grad} \varphi, \operatorname{div} \vec{v}=0 ;(x, y, z) \in G_{\mathbf{z}}^{r}, r=\overline{1, p},
$$




$$
\left\{\begin{array}{l}
(\sigma \cdot C)_{t}^{\prime}=\operatorname{div}(D \cdot \operatorname{grad} C)-\vec{v} \cdot \operatorname{grad} C- \\
-\alpha \cdot C+\beta \cdot U-\alpha^{*} \cdot C \\
(\sigma \cdot U)^{\prime}=\operatorname{div}\left(D^{*} \cdot \operatorname{grad} U\right)+\alpha \cdot C-\beta \cdot U ; \\
(\sigma \cdot W)^{\prime}=\operatorname{div}\left(D^{* *} \cdot \operatorname{grad} W\right)+\alpha^{*} \cdot C ; \\
(\sigma \cdot T)^{\prime}=\operatorname{div}\left(D^{* * *} \cdot \operatorname{grad} T\right)-\vec{v} \cdot \operatorname{grad} T+ \\
+\gamma \cdot(\alpha \cdot C-\beta \cdot U)+\gamma^{*} \cdot \alpha^{*} \cdot C ; \\
\kappa_{t}^{\prime}=-\mu \cdot U-\mu^{*} \cdot W ; \\
\sigma_{t}^{\prime}=-\lambda \cdot U-\lambda^{*} \cdot W ;(x, y, z) \in G_{\mathbf{z}}^{r}, r=\overline{1, p}
\end{array}\right.
$$

які доповнюються наступними крайовими і початковими умовами

$$
\begin{aligned}
& \left.\varphi\right|_{S_{*}}=\varphi_{*},\left.\varphi\right|_{S^{*}}=\varphi^{*},\left.\varphi_{\vec{n}}^{\prime}\right|_{S^{* *}}=0, \\
& \left\{\begin{array}{l}
\left.C\right|_{S_{*}}=c_{*}^{*},\left.C_{\vec{n}}^{\prime}\right|_{S^{*}}=0,\left.C_{\vec{n}}^{\prime}\right|_{S^{* *}}=0 ; \\
\left.U\right|_{S_{*}}=u_{*}^{*},\left.U_{\vec{n}}^{\prime}\right|_{S^{*}}=0,\left.U_{\vec{n}}^{\prime}\right|_{S^{* *}}=0 ; \\
\left.W\right|_{S_{*}}=u_{*}^{*},\left.W_{\vec{n}}^{\prime}\right|_{S^{*}}=0,\left.W_{\vec{n}}^{\prime}\right|_{S^{* *}}=0 ; \\
\left.T\right|_{S_{*}}=T_{*}^{*},\left.T_{\vec{n}}^{\prime}\right|_{S^{*}}=0,\left.T_{\vec{n}}^{\prime}\right|_{S^{* *}}=0,
\end{array}\right. \\
& \left\{\begin{array}{l}
\left.C\right|_{t=0}=c_{0}^{0},\left.\quad U\right|_{t=0}=u_{0}^{0},\left.\quad W\right|_{t=0}=w_{0}^{0} ; \\
\left.T\right|_{t=0}=T_{0}^{0},\left.\quad \kappa\right|_{t=0}=\kappa_{0}^{0},\left.\quad \sigma\right|_{t=0}=\sigma_{0}^{0}
\end{array}\right.
\end{aligned}
$$

та умовами узгодженості на поверхнях розділу $S_{* r}^{*}(r=\overline{1, p-1})$

$$
\begin{aligned}
& \left.\varphi\right|_{S_{r-}}=\left.\varphi\right|_{S_{r+}}=\varphi_{* r}^{*},\left.\quad \kappa_{r}^{0} \cdot \varphi_{\vec{n}}^{\prime}\right|_{S_{r-}}=\left.\kappa_{r}^{0} \cdot \varphi_{\vec{n}}^{\prime}\right|_{S_{r+}}, r=\overline{1, p-1}, \\
& \left\{\begin{array}{l}
\left.C\right|_{S_{r-}}=\left.C\right|_{S_{r+}}, \quad D_{r} \cdot C_{\vec{n}}^{\prime}-\left.v_{r n}^{0} \cdot C\right|_{S_{r-}}=D_{r+1} \cdot C_{\vec{n}}^{\prime}-\left.v_{r n}^{0} \cdot C\right|_{S_{r+}} ; \\
\left.U\right|_{S_{r-}}=\left.U\right|_{S_{r+}},\left.D_{r}^{*} \cdot U_{\vec{n}}^{\prime}\right|_{S_{r-}}=\left.D_{r+1}^{*} \cdot U_{\vec{n}}^{\prime}\right|_{S_{r+}} \\
\left.W\right|_{S_{r-}}=\left.W\right|_{S_{r+}},\left.D_{r}^{* *} \cdot W_{\vec{n}}^{\prime}\right|_{S_{r-}}=\left.D_{r+1}^{* *} \cdot W_{\vec{n}}^{\prime}\right|_{S_{r+}} ; \\
\left.T\right|_{S_{r-}}=T-\left.\theta \cdot T\right|_{S_{r+}}, \quad D_{r}^{* * *} \cdot T_{\vec{n}}^{\prime}-\left.v_{r n}^{0} \cdot T\right|_{S_{r-}}=
\end{array}\right. \\
& =D_{r+1}^{* * *} \cdot T_{\vec{n}}^{\prime}-v_{r n}^{0} \cdot T-\left.\theta \cdot T\right|_{S_{r+}} ; \\
& D_{r} \cdot C_{\vec{n}}^{\prime}-v_{r n} \cdot C+D_{r}^{*} \cdot U^{\prime}+\left.D_{r}^{* *} \cdot W_{\vec{n}}^{\prime}\right|_{S_{r-}}= \\
& =D_{r+1, j} \cdot C^{\prime}{ }_{j \vec{n}}-v_{r n} \cdot C_{j}+D_{r+1}^{*} \cdot U_{\vec{n}}^{\prime}+\left.D_{r+1}^{* *} \cdot W_{\vec{n}}^{\prime}\right|_{S_{r+}} ; \\
& \left.(\sigma \cdot(C+U+W))^{\prime}\right|_{S_{r_{-}}}=\left.(\sigma \cdot(C+U+W))_{t}{ }_{t}\right|_{S_{r+}}, r=\overline{1, p-1},
\end{aligned}
$$

де $\varphi=\varphi(x, y, z)$ i $\vec{v}=\vec{v}\left(v_{x}, v_{y}, v_{z}\right)$ - відповідно потенціал і вектор швидкості фільтрації, $0 \leqslant \varphi_{*}=\varphi_{* 0}^{*}<\varphi_{* 1}^{*}<\ldots<\varphi_{* p-1}^{*}<\varphi_{* p}^{*}=\varphi^{*}<\infty, \varphi_{*}$ i $\varphi^{*}-$ задані дійсні числа, $\varphi_{* 1}^{*}, \varphi_{* 2}^{*}, \ldots, \varphi_{*(p-1)}^{*}$ - невідомі значення потенціалу, $v=|\vec{v}|=\sqrt{v_{x}^{2}(x, y, z)+v_{y}^{2}(x, y, z)+v_{z}^{2}(x, y, z)}>>0, \kappa_{0}^{0}$ - початковий коефіцієнт фільтрації, $\kappa_{0}^{0}=\left\{\kappa_{r}^{0},(x, y, z) \in G_{z}^{r}, r=\overline{1, p}\right\}, \kappa_{r}^{0}>0(r=\overline{1, p}), \vec{n}-$ зовнішня нормаль до відповідної поверхні, $C=C(x, y, z, t), U=U(x, y, z, t)$ і $W=W(x, y, z, t)$ - концентрації домішок відповідно у фільтраційній течіï та адсорбованих на поверхні адсорбенту завантаження при фізичній i 
хімічній адсорбції, $T=T(x, y, z, t)$ - температура в точці $(x, y, z)$ у момент часу $t, \kappa=\kappa(x, y, z, t)$ - коефіцієнт фільтрації, $\sigma=\sigma(x, y, z, t)$ - активна пористість, $D, D^{*}$ і $D^{* *}$ - коефіцієнти дифузії домішок відповідно у фільтраційній течії та адсорбованих на поверхні адсорбенту завантаження відповідно при фізичній і хімічній адсорбції, $D=\left\{D_{r},(x, y, z) \in G_{\mathbf{z}}^{r}, r=\right.$ $=\overline{1, p}\}, D_{r}=\varepsilon \cdot d_{r, 0}, d_{r, 0}>0(r=\overline{1, p}), D^{*}=\left\{D_{r}^{*},(x, y, z) \in G_{\mathbf{z}}^{r}, r=\overline{1, p}\right\}$, $D_{r}^{*}=\varepsilon \cdot d_{r, 0}^{*}, d_{r, 0}^{*}>0(r=\overline{1, p}), D^{* *}=\left\{D_{r}^{* *},(x, y, z) \in G_{\mathbf{z}}^{r}, r=\overline{1, p}\right\}$, $D_{r}^{* *}=\varepsilon \cdot d_{r, 0}^{* *}, d_{r, 0}^{* *}>0(r=\overline{1, p}), D^{* * *}$ - коефіцієнт температуропровідності, $D^{* * *}=\left\{D_{r}^{* * *},(x, y, z) \in G_{\mathbf{z}}^{r}, r=\overline{1, p}\right\}, D_{r}^{* * *}=\varepsilon \cdot d_{r, 0}^{* * *}, d_{r, 0}^{* * *}>0(r=\overline{1, p}), \alpha \mathrm{i}$ $\beta$ - коефіцієнти, що характеризують швидкість масообміну відповідно при фізичній адсорбції та десорбції домішок, $\alpha=\left\{\alpha_{r},(x, y, z) \in G_{\mathbf{z}}^{r}, r=\overline{1, p}\right\}$, $\alpha_{r}=\alpha_{r, 0,0}+\varepsilon \cdot \alpha_{r, 1,0} \cdot v+\varepsilon^{2} \cdot \alpha_{r, 2,0} \cdot v^{2}+\varepsilon \cdot \alpha_{r, 0,1} \cdot T+\varepsilon^{2} \cdot \alpha_{r, 0,2} \cdot T^{2}+\varepsilon^{2} \cdot \alpha_{r, 2,2} \cdot v \cdot T$, $\alpha_{r, s_{1}, s_{2}} \in \mathrm{R}\left(r=\overline{1, p}, s_{1}=\overline{0,2}, s_{2}=\overline{0,2}\right), \beta=\left\{\beta_{r},(x, y, z) \in G_{\mathbf{z}}^{r}, r=\overline{1, p}\right\}$, $\beta_{r}=\varepsilon \cdot \bar{\beta}_{r}, \bar{\beta}_{r}=\beta_{r, 0,0}+\varepsilon \cdot \beta_{r, 1,0} \cdot v+\varepsilon^{2} \cdot \beta_{r, 2,0} \cdot v^{2}+\varepsilon \cdot \beta_{r, 0,1} \cdot T+\varepsilon^{2} \cdot \beta_{r, 0,2} \cdot T^{2}+$ $+\varepsilon^{2} \cdot \beta_{r, 2,2} \cdot v \cdot T, \beta_{r, s_{1}, s_{2}} \in \mathrm{R}\left(r=\overline{1, p}, s_{1}=\overline{0,2}, s_{2}=\overline{0,2}\right), \alpha^{*}$ - коефіцієнт, що характеризуе швидкість масообміну при хімічній адсорбції домішок, $\alpha^{*}=\alpha_{0,0}^{*}+\varepsilon \cdot \alpha_{1,0}^{*} \cdot v+\varepsilon^{2} \cdot \alpha_{2,0}^{*} \cdot v^{2}+\varepsilon \cdot \alpha_{0,1}^{*} \cdot T+\varepsilon^{2} \cdot \alpha_{0,2}^{*} \cdot T^{2}+\varepsilon^{2} \cdot \alpha_{2,2}^{*} \cdot v \cdot T, \alpha_{s_{1}, s_{2}}^{*} \in \mathrm{R}$ $\left(s_{1}=\overline{0,2}, s_{2}=\overline{0,2}\right), \gamma$ i $\gamma^{*}$ - коефіцієнти, що характеризують швидкість зміни температури за рахунок відповідно фізичної і хімічної адсорбції домішок, $\gamma=\left\{\gamma_{r},(x, y, z) \in G_{\mathbf{z}}^{r}, r=\overline{1, p}\right\}, \gamma^{*}=\left\{\gamma_{r}^{*},(x, y, z) \in G_{\mathbf{z}}^{r}, r=\overline{1, p}\right\}$, $\mu, \lambda, \mu^{*}$ і $\lambda^{*}$ - коефіцієнти, що характеризують відповідно швидкість зміни коефіцієнтів фільтрації й активної пористості завантаження за рахунок відповіднл фізичної і хімічної адсорбції домішок, $\mu=\left\{\mu_{r},(x, y, z) \in G_{\mathbf{z}}^{r}\right.$, $r=\overline{1, p}\}, \mu_{r}=\varepsilon \cdot \bar{\mu}_{r}, \bar{\mu}_{r}=\mu_{r, 0}+\varepsilon \cdot \mu_{r, 1} \cdot T+\varepsilon^{2} \cdot \mu_{r, 2} \cdot T^{2}, \mu_{r, s} \in \mathrm{R}$ $(r=\overline{1, p}, s=\overline{0,2}), \lambda=\left\{\lambda_{r},(x, y, z) \in G_{\mathbf{z}}^{r}, r=\overline{1, p}\right\}, \lambda_{r}=\varepsilon \cdot \bar{\lambda}_{r}(r=\overline{1, p})$, $\mu^{*}=\left\{\mu_{r}^{*},(x, y, z) \in G_{\mathbf{z}}^{r}, r=\overline{1, p}\right\}, \mu_{r}^{*}=\varepsilon \cdot \bar{\mu}_{r}^{*}, \bar{\mu}_{r}^{*}=\mu_{r, 0}^{*}+\varepsilon \cdot \mu_{r, 1}^{*} \cdot T+\varepsilon^{2} \cdot \mu_{r, 2}^{*} \cdot T^{2}$, $\mu_{r, s}^{*} \in \mathrm{R}(r=\overline{1, p}, s=\overline{0,2}), \lambda^{*}=\left\{\lambda_{r}^{*},(x, y, z) \in G_{\mathbf{z}}^{r}, r=\overline{1, p}\right\}, \lambda_{r}^{*}=\varepsilon \cdot \bar{\lambda}_{r}^{*}$ $(r=\overline{1, p}), \theta_{r}$ - коефіцієнти, що характеризують швидкість зміни температури на поверхнях розділу $S_{* r}^{*}(r=\overline{1, p-1})$ за рахунок відведення частини тепла, $\alpha_{r}=\alpha_{r}(x, y, z, t), \bar{\beta}_{r}=\bar{\beta}_{r}(x, y, z, t), \alpha_{r}^{*}=\alpha_{r}^{*}(x, y, z, t)$, $\gamma_{r}=\gamma_{r}(x, y, z, t), \gamma_{r}^{*}=\gamma_{r}^{*}(x, y, z, t), \bar{\mu}_{r}=\bar{\mu}_{r}(x, y, z, t), \bar{\lambda}_{r}=\bar{\lambda}_{r}(x, y, z, t)$, $\bar{\mu}_{r}^{*}=\bar{\mu}_{r}^{*}(x, y, z, t), \bar{\lambda}_{r}^{*}=\bar{\lambda}_{r}^{*}(x, y, z, t), \theta_{r}=\theta_{r}(x, y, z)$ - неперервні обмежені функції $(r=\overline{1, p}), \varepsilon$ - малий параметр $(\varepsilon>0), c_{0}^{0}=\left\{c_{r}^{0},(x, y, z) \in G_{\mathbf{z}}^{r}, r=\right.$ $=\overline{1, p}\}, u_{0}^{0}=\left\{u_{r}^{0},(x, y, z) \in G_{\mathbf{z}}^{r}, r=\overline{1, p}\right\}, w_{0}^{0}=\left\{w_{r}^{0},(x, y, z) \in G_{\mathbf{z}}^{r}, r=\overline{1, p}\right\}$, $T_{0}^{0}=\left\{T_{r}^{0},(x, y, z) \in G_{\mathbf{z}}^{r}, r=\overline{1, p}\right\}, \sigma_{0}^{0}=\left\{\sigma_{r}^{0},(x, y, z) \in G_{\mathbf{z}}^{r}, r=\overline{1, p}\right\}$, $c_{*}^{*}=c_{*}^{*}(M, t), c_{r}^{0}=c_{r}^{0}(x, y, z), u_{*}^{*}=u_{*}^{*}(M, t), u_{r}^{0}=u_{r}^{0}(x, y, z), w_{*}^{*}=w_{*}^{*}(M, t)$, $w_{r}^{0}=w_{r}^{0}(x, y, z), T_{*}^{*}=T_{*}^{*}(M, t), T_{r}^{0}=T_{r}^{0}(x, y, z), \sigma_{r}^{0}=\sigma_{r}^{0}(x, y, z)(r=\overline{1, p})$ - достатньо гладкі функції, узгоджені між собою на ребрах області $G$ [16], $M \in S_{*}, v_{r n}^{0}$ i $v_{r n}(r=\overline{1, p-1})$ - відповідно початкові і поточні нормальні складові швидкості на поверхнях розділу $S_{* r}^{*}(r=\overline{1, p-1})$.

Аналогічно до [16] шляхом виконання умовного розрізу $\Gamma=\Gamma_{1} \cup \Gamma_{2}$, $\Gamma_{1}=A L M D B L M C$ i $\Gamma_{2}=A D D_{*} A_{*} B C C_{*} B_{*}$, задача (1)-(7) зводиться до розв'язання в однозв'язній кусково-однорідній області $G_{z} \backslash \Gamma$ - криволінійному паралелепіпеді $A B C D A_{*} B_{*} C_{*} D_{*}$, обмеженому двома еквіпотенціальними поверхнями $A B B_{*} A_{*}, C D D_{*} C_{*}$ і чотирма поверхнями течії $A B C D=$ 
$=A L M D \cup B L M C, A_{*} B_{*} C_{*} D_{*}, A D D_{*} A_{*}=B C C_{*} B_{*}$ та розділеному еквіпотенціальними поверхнями $E_{r} F_{r} F_{* r} E_{* r}(r=\overline{1, p-1})$ на $p$ підобластей $G_{\mathbf{z}}^{1} \backslash \Gamma^{1}=A B F_{1} E_{1} A_{*} B_{*} F_{* 1} E_{* 1}, \ldots, G_{\mathbf{z}}^{p} \backslash \Gamma^{p}=E_{p-1} F_{p-1} C D E_{*(p-1)} F_{*(p-1)} C_{*} D_{*}$ $\left(\Gamma^{1}=A E_{1} E_{* 1} A_{*} B F_{1} F_{* 1} B_{*}, \ldots, \Gamma^{p}=E_{p-1} D D_{*} E_{*(p-1)} F_{p-1} C C_{*} F_{*(p-1)}\right)$, які є гладкими і ортогональними між собою в кутових точках та вздовж ребер (рис. 1 а), з додаванням умови непроникності $\left.\varphi_{\vec{n}}^{\prime}\right|_{\Gamma}=0$ вздовж розрізу $\Gamma$ задачі, що описується системами рівнянь (1), (2) з крайовими умовами

$$
\begin{aligned}
& \left\{\begin{array}{l}
\left.\varphi\right|_{A B B_{*} A_{*}}=\varphi_{*},\left.\varphi\right|_{C D D_{*} C_{*}}=\varphi^{*} ; \\
\left.\varphi^{\prime}\right|_{A B C D \cup A_{*} B_{*} C_{*} D_{*} \cup A D D_{*} A_{*} \cup B C C_{*} B_{*}}=0,
\end{array}\right. \\
& \left\{\begin{array}{l}
\left.C\right|_{A B B_{*} A_{*}}=c_{*}^{*},\left.C_{\vec{n}}^{\prime}\right|_{C D D_{*} C_{*}}=0 ; \\
\left.C_{\vec{n}}^{\prime}\right|_{A D D_{*} A_{*} \cup B C C_{*} B_{*} \cup A B C D \cup A_{*} B_{*} C_{*} D_{*}}=0 ; \\
\left.U\right|_{A B B_{*} A_{*}}=u_{*}^{*},\left.U_{\vec{n}}^{\prime}\right|_{C D D_{*} C_{*}}=0 ; \\
\left.U^{\prime}\right|_{A D D_{*} A_{*} \cup B C C_{*} B_{*} \cup A B C D \cup A_{*} B_{*} C_{*} D_{*}}=0 ; \\
\left.W\right|_{A B B_{*} A_{*}}=w_{*}^{*},\left.W_{\vec{n}}^{\prime}\right|_{C D D_{*} C_{*}}=0 ; \\
\left.W_{\vec{n}}^{\prime}\right|_{A D D_{*} A_{*} \cup B C C_{*} B_{*} \cup A B C D \cup A_{*} B_{*} C_{*} D_{*}=0 ;} \\
\left.T\right|_{A B B_{*} A_{*}}=T_{*}^{*},\left.T_{\vec{n}}^{\prime}\right|_{C D D_{*} C_{*}}=0 ; \\
\left.T_{\vec{n}}^{\prime}\right|_{A D D_{*} A_{*} \cup B C C_{*} B_{*} \cup A B C D \cup A_{*} B_{*} C_{*} D_{*}}=0,
\end{array}\right.
\end{aligned}
$$

початковими умовами (5), умовами узгодженості на поверхнях розділу $E_{r} F_{r} F_{* r} E_{* r}(r=\overline{1, p-1})$

$$
\begin{aligned}
& \left\{\begin{array}{l}
\left.\varphi\right|_{E_{r} F_{r} F_{* r} E_{* r-}}=\left.\varphi\right|_{E_{r} F_{r} F_{* r} E_{* r+}}=\varphi_{* r}^{*} ; \\
\left.\kappa_{r}^{0} \cdot \varphi_{\vec{n}}^{\prime}\right|_{E_{r} F_{r} F_{* r} E_{* r-}}=\left.\kappa_{r+1}^{0} \cdot \varphi_{\vec{n}}^{\prime}\right|_{E_{r} F_{r} F_{* r} E_{* r+}}, r=\overline{1, p-1},
\end{array}\right. \\
& \left\{\begin{array}{l}
\left.C\right|_{E_{r} F_{r} F_{* r} E_{* r-}}=\left.C\right|_{E_{r} F_{r} F_{* r} E_{* r+}+} ; \\
D_{r} \cdot C_{\vec{n}}^{\prime}-\left.v_{r n}^{0} \cdot C\right|_{E_{r} F_{r} F_{* r} E_{* r-}}=D_{r+1} \cdot C^{\prime}-\left.v_{r n}^{0} \cdot C\right|_{E_{r} F_{r} F_{* r} E_{* r+}} ;
\end{array}\right. \\
& \left.U\right|_{E_{r} F_{r} F_{* r} E_{* r-}}=\left.U\right|_{E_{r} F_{r} F_{* r} E_{* r+}} \text {; } \\
& \left.D_{r}^{*} \cdot U_{\vec{n}}^{\prime}\right|_{E_{r} F_{r} F_{* r} E_{* r-}}=\left.D_{r+1}^{*} \cdot U_{\vec{n}}^{\prime}\right|_{E_{r} F_{r} F_{* r} E_{* r+}} \text {; } \\
& \left.W\right|_{E_{r} F_{r} F_{* r} E_{* r-}}=\left.W\right|_{E_{r} F_{r} F_{* r} E_{* r+}} ; \\
& \left.D_{r}^{* *} \cdot W_{\vec{n}}^{\prime}\right|_{E_{r} F_{r} F_{* r} E_{* r-}}=\left.D_{r+1}^{* *} \cdot W_{\vec{n}}^{\prime}\right|_{E_{r} F_{r} F_{* r} E_{* r+}} ; \\
& \left.T\right|_{E_{r} F_{r} F_{* r} E_{* r-}}=T-\left.\theta_{r} \cdot T\right|_{E_{r} F_{r} F_{* r} E_{* r+}} ; \\
& D_{r}^{* * *} \cdot T_{\vec{n}}^{\prime}-\left.v_{r n}^{0} \cdot T\right|_{E_{r} F_{r} F_{* r} E_{* r-}}= \\
& =D_{r+1}^{* * *} \cdot T_{\vec{n}}^{\prime}-v_{r n}^{0} \cdot T-\left.\theta_{r} \cdot T\right|_{E_{r} F_{r} F_{* r} E_{* r+}} ; \\
& D_{r} \cdot C_{\vec{n}}^{\prime}-v_{r n} \cdot C+D_{r}^{*} \cdot U_{\vec{n}}^{\prime}+\left.D_{r}^{* *} \cdot W_{\vec{n}}^{\prime}\right|_{E_{r} F_{r} F_{* r} E_{* r-}}= \\
& =D_{r+1} \cdot C_{\vec{n}}^{\prime}-v_{r n} \cdot C+D_{r+1}^{*} \cdot U_{\vec{n}}^{\prime}+\left.D_{r+1}^{* *} \cdot W_{n}^{\prime}\right|_{E_{r} F_{r} F_{* r} E_{* r+}} ; \\
& \left.(\sigma \cdot(C+U+W))^{\prime}{ }_{t}\right|_{E_{r} F_{r} F_{* r} E_{* r-}}= \\
& \begin{array}{l}
=\left.(\sigma \cdot(C+U+W))^{\prime}{ }_{t}\right|_{E_{r} F_{r} F_{* r} E_{* r+}} ; \\
r=\overline{1, p-1}
\end{array}
\end{aligned}
$$


умовами „склеювання“ берегів умовного розрізу $Г$ і узгодженості величин концентрацій домішок та температури на умовних поверхнях розрізу $\Gamma$

$$
\begin{gathered}
\left\{\begin{array}{l}
\left.\varphi\right|_{A L M D}=\left.\varphi\right|_{B L M C},\left.\varphi_{\vec{n}}^{\prime}\right|_{A L M D}=\left.\varphi_{\vec{n}}^{\prime}\right|_{B L M C} ; \\
\left.\varphi\right|_{A D D_{*} A_{*}}=\left.\varphi\right|_{B C C_{*} B_{*}},\left.\varphi_{\vec{n}}^{\prime}\right|_{A D D_{*} A_{*}}=\left.\varphi_{\vec{n}}^{\prime}\right|_{B C C_{*} B_{*}}
\end{array}\right. \\
\left\{\begin{array}{l}
\left.C\right|_{A L M D}=\left.C\right|_{B L M C},\left.C_{\vec{n}}^{\prime}\right|_{A L M D}=\left.C_{\vec{n}}^{\prime}\right|_{B L M C} ; \\
\left.C\right|_{A D D_{*} A_{*}}=\left.C\right|_{B C C_{*} B_{*}},\left.C_{\vec{n}}^{\prime}\right|_{A D D_{*} A_{*}}=\left.C_{\vec{n}}^{\prime}\right|_{B C C_{*} B_{*}} ; \\
\left.U\right|_{A L M D}=\left.U\right|_{B L M C},\left.U_{\vec{n}}^{\prime}\right|_{A L M D}=\left.U_{\vec{n}}^{\prime}\right|_{B L M C} ; \\
\left.U\right|_{A D D_{*} A_{*}}=\left.U\right|_{B C C_{*} B_{*}},\left.U_{\vec{n}}^{\prime}\right|_{A D D_{*} A_{*}}=\left.U_{\vec{n}}^{\prime}\right|_{B C C_{*} B_{*}} ; \\
\left.W\right|_{A L M D}=\left.W\right|_{B L M C},\left.W_{\vec{n}}^{\prime}\right|_{A L M D}=\left.W_{\vec{n}}^{\prime}\right|_{B L M C} ; \\
\left.W\right|_{A D D_{*} A_{*}}=\left.W\right|_{B C C_{*} B_{*}},\left.W_{\vec{n}}^{\prime}\right|_{A D D_{*} A_{*}}=\left.W_{\vec{n}}^{\prime}\right|_{B C C_{*} B_{*}} ; \\
\left.T\right|_{A L M D}=\left.T\right|_{B L M C},\left.T_{\vec{n}}^{\prime}\right|_{A L M D}=\left.T_{\vec{n}}^{\prime}\right|_{B L M C} ; \\
\left.T\right|_{A D D_{*} A_{*}}=\left.T\right|_{B C C_{*} B_{*}},\left.T_{\vec{n}}^{\prime}\right|_{A D D_{*} A_{*}}=\left.T_{\vec{n}}^{\prime}\right|_{B C C_{*} B_{*}}
\end{array}\right.
\end{gathered}
$$

Аналогічно [16] задача (1), (8), (10), (12) замінюється більш загальною прямою задачею знаходження просторового аналогу кусково-конформного відображення однозв'язної області $G_{\mathbf{z}} \backslash \Gamma$ на відповідну область комплексного потенціалу - прямокутний паралелепіпед $G_{\mathbf{w}}=A^{\prime} B^{\prime} C^{\prime} D^{\prime} A_{*}^{\prime} B_{*}^{\prime} C_{*}^{\prime} D_{*}^{\prime}$, який розділений поверхнями $E_{r}^{\prime} F_{r}^{\prime} E_{* r}^{\prime} F_{* r}^{\prime}(r=\overline{1, p})$ на деякі $p$ підобластей $G_{\mathbf{w}}^{1}=A^{\prime} B^{\prime} E_{1}^{\prime} F_{1}^{\prime} A_{*}^{\prime} B_{*}^{\prime} E_{* 1}^{\prime} F_{* 1}^{\prime}, G_{\mathbf{w}}^{r}=E_{r}^{\prime} F_{r}^{\prime} F_{r+1}^{\prime} E_{r+1}^{\prime} E_{* r}^{\prime} F_{* r}^{\prime} F_{* r+1}^{\prime} E_{* r+1}^{\prime}$ $(r=\overline{2, p-2}), G_{\mathbf{w}}^{p}=E_{p-1}^{\prime} F_{p-1}^{\prime} C^{\prime} D^{\prime} E_{* p-1}^{\prime} F_{* p-1}^{\prime} C_{*}^{\prime} D_{*}^{\prime}$ (рис. 1, б), де $G_{\mathbf{w}}^{r}=$ $\left\{\mathbf{w}=(\varphi, \psi, \eta): \varphi_{*(r-1)}^{*}<\varphi<\varphi_{* r}^{*}, 0<\psi<Q_{*}, 0<\eta<Q^{*}\right\}(r=\overline{1, p})$, $\varphi_{* r}^{*}(r=\overline{1, p-1}), Q_{*}, Q^{*}$ - невідомі параметри, $Q=Q_{*} \cdot Q^{*}-$ повна фільтраційна витрата, і наступного „склеювання“ берегів умовного розрізу $Г$. Алгоритм розв'язання цієї задачі отримано в [17], зокрема, знайдено поле швидкостей $\vec{v}$, параметри $Q_{*}, Q^{*}, Q$ та ряд інших величин. Задача $(2),(9)$, $(5),(10),(11),(13)$ для області $G_{\mathrm{w}} \times(0, \infty)$ матиме вигляд

$$
\left\{\begin{array}{l}
(\tilde{\sigma} \cdot c)^{\prime}{ }_{t}=D \cdot\left(b_{1} \cdot c^{\prime \prime}{ }_{\varphi \varphi}+b_{2} \cdot c^{\prime \prime}{ }_{\psi \psi}+b_{3} \cdot c^{\prime \prime}{ }_{\eta \eta}+b_{4} \cdot c^{\prime}{ }_{\psi}+b_{5} \cdot c^{\prime}{ }_{\eta}\right)- \\
-\frac{\tilde{v}^{2}}{\kappa} \cdot c^{\prime}{ }_{\varphi}-\tilde{\alpha} \cdot c+\tilde{\beta} \cdot u-\tilde{\alpha}^{*} \cdot c ; \\
(\tilde{\sigma} \cdot u)^{\prime}=D^{*} \cdot\left(b_{1} \cdot u^{\prime \prime}{ }_{\varphi \varphi}+b_{2} \cdot u^{\prime \prime}{ }_{\psi \psi}+b_{3} \cdot u^{\prime \prime}{ }_{\eta \eta}+b_{4} \cdot u_{\psi}^{\prime}+b_{5} \cdot u_{\eta}^{\prime}\right)+ \\
+\tilde{\alpha} \cdot c-\tilde{\beta} \cdot u ; \\
(\tilde{\sigma} \cdot w)^{\prime}{ }_{t}=D^{* *} \cdot\left(b_{1} \cdot w^{\prime \prime}{ }_{\varphi \varphi}+b_{2} \cdot w^{\prime \prime}{ }_{\psi \psi}+b_{3} \cdot w^{\prime \prime}{ }_{\eta \eta}+b_{4} \cdot w^{\prime}{ }_{\psi}+\right. \\
\left.+b_{5} \cdot w^{\prime}{ }_{\eta}\right)+\tilde{\alpha}^{*} \cdot c ; \\
(\tilde{\sigma} \cdot \tilde{T})^{\prime}{ }_{t}=D^{* *} \cdot\left(b_{1} \cdot \tilde{T}_{\varphi \varphi}^{\prime \prime}+b_{2} \cdot \tilde{T}_{\psi \psi}^{\prime \prime}+b_{3} \cdot \tilde{T}_{\eta \eta}^{\prime \prime}+b_{4} \cdot \tilde{T}_{\psi}^{\prime}+b_{5} \cdot \tilde{T}_{\eta}^{\prime}\right)- \\
-\frac{\tilde{v}^{2}}{\kappa} \cdot \tilde{T}_{\varphi}^{\prime}+\tilde{\gamma} \cdot(\tilde{\alpha} \cdot c-\tilde{\beta} \cdot u)+\tilde{\gamma}^{*} \cdot \tilde{\alpha}^{*} \cdot c ; \\
\tilde{\kappa}_{t}^{\prime}=-\tilde{\mu} \cdot u-\tilde{\mu}^{*} \cdot w ; \\
\tilde{\sigma}_{t}^{\prime}=-\tilde{\lambda} \cdot u-\tilde{\lambda}^{*} \cdot w ;(\varphi, \psi, \eta) \in G_{\mathbf{w}}^{r}, r=\overline{1, p},
\end{array}\right.
$$




$$
\begin{aligned}
& \left\{\begin{array}{l}
\left.c\right|_{\varphi=\varphi_{*}}=\tilde{c}_{*}^{*},\left.c_{\varphi}^{\prime}\right|_{\varphi=\varphi^{*}}=0 ; \\
\left.c_{\psi}\right|_{\psi=0}=c_{\psi}^{\prime}{ }_{\psi=Q_{*}}=\left.c_{\eta}^{\prime}\right|_{\eta=0}=\left.c^{\prime}\right|_{\eta=Q^{*}}=0 ; \\
\left.u\right|_{\varphi=\varphi_{*}}=\tilde{u}_{*}^{*},\left.u_{\varphi}^{\prime}\right|_{\varphi=\varphi^{*}}=0 ; \\
\left.u_{\psi}^{\prime}\right|_{\psi=0}=\left.u_{\psi}^{\prime}\right|_{\psi=Q_{*}}=\left.u_{\eta}^{\prime}\right|_{\eta=0}=\left.u_{\eta}^{\prime}\right|_{\eta=Q^{*}}=0 ; \\
\left.w\right|_{\varphi=\varphi_{*}}=\tilde{w}_{*}^{*},\left.w_{\varphi}^{\prime}\right|_{\varphi=\varphi^{*}}=0 ; \\
\left.w_{\psi}^{\prime}\right|_{\psi=0}=\left.w_{\psi}^{\prime}\right|_{\psi=Q_{*}}=\left.w_{\eta}^{\prime}\right|_{\eta=0}=\left.w_{\eta}^{\prime}\right|_{\eta=Q^{*}}=0 ; \\
\left.\tilde{T}\right|_{\varphi=\varphi_{*}}=\tilde{T}_{*}^{*},\left.\tilde{T}_{\varphi}^{\prime}\right|_{\varphi=\varphi^{*}}=0 ; \\
\left.\tilde{T}_{\psi}^{\prime}\right|_{\psi=0}=\left.\tilde{T}_{\psi}^{\prime}\right|_{\psi=Q_{*}}=\left.\tilde{T}_{\eta}^{\prime}\right|_{\eta=0}=\left.\tilde{T}_{\eta}^{\prime}\right|_{\eta=Q^{*}}=0,
\end{array}\right. \\
& \left\{\begin{array}{l}
\left.c\right|_{t=0}=\tilde{c}_{0}^{0},\left.u\right|_{t=0}=\tilde{u}_{0}^{0},\left.w\right|_{t=0}=\tilde{w}_{0}^{0} \\
\left.\tilde{T}\right|_{t=0}=\tilde{T}_{0}^{0},\left.\tilde{\kappa}\right|_{t=0}=\kappa_{0}^{0},\left.\tilde{\sigma}\right|_{t=0}=\tilde{\sigma}_{0}^{0}
\end{array}\right.
\end{aligned}
$$

$$
\begin{aligned}
& \left\{\begin{array}{l}
\lim _{\varphi \rightarrow \varphi_{* r-0}^{*}} c=\lim _{\varphi \rightarrow \varphi_{* r+0}^{*}} c ; \\
\lim _{\varphi \rightarrow \varphi_{* r-0}^{*}}\left(D_{r} \cdot c_{\varphi}^{\prime}-\kappa_{r}^{0} \cdot c\right)=\lim _{\varphi \rightarrow \varphi_{* r+0}^{*}}\left(D_{r+1} \cdot c_{\varphi}^{\prime}-\kappa_{r+1}^{0} \cdot c\right) ; \\
\lim _{\varphi \rightarrow \varphi_{* r-0}^{*}} u=\lim _{\varphi \rightarrow \varphi_{* r+0}^{*}} u, \lim _{\varphi \rightarrow \varphi_{* r-0}^{*}} D_{r}^{*} \cdot u_{\varphi}^{\prime}=\lim _{\varphi \rightarrow \varphi_{* r+0}^{*}} D_{r+1}^{*} \cdot u_{\varphi}^{\prime} ; \\
\lim _{\varphi \rightarrow \varphi_{* r-0}^{*}} w=\lim _{\varphi \rightarrow \varphi_{* r+0}^{*}} w, \lim _{\varphi \rightarrow \varphi_{* r-0}^{*}} D_{r}^{* *} \cdot w_{\varphi}^{\prime}=\lim _{* \rightarrow+0}^{*} D_{r+1}^{* *} \cdot w_{\varphi}^{\prime} ; \\
\lim _{\varphi \rightarrow \varphi_{* r-0}^{*}} \tilde{T}=\lim _{\varphi \rightarrow \varphi_{* r+0}^{*}}(\tilde{T}-\tilde{\theta} \cdot T) ; \\
\lim _{\varphi \rightarrow \varphi_{* r-0}^{*}}\left(D_{r}^{* * *} \cdot \tilde{T}_{\varphi}^{\prime}-\kappa_{r}^{0} \cdot \tilde{T}\right)=\lim _{\varphi \rightarrow \varphi_{* r+0}^{*}}\left(D_{r+1}^{* * *} \cdot \tilde{T}_{\varphi}^{\prime}-\kappa_{r+1}^{0} \cdot \tilde{T}-\tilde{\theta} \cdot T\right) ; \\
\lim _{\varphi \rightarrow \varphi_{* r-0}^{*}}\left(D_{r} \cdot c^{\prime}{ }_{\varphi}-\tilde{\kappa}_{r} \cdot c+D_{r}^{*} \cdot u_{\varphi}^{\prime}+D_{r}^{* *} \cdot w_{\varphi}^{\prime}\right)= \\
=\lim _{\varphi \rightarrow \varphi_{* r+0}^{*}}\left(D_{r+1} \cdot c_{\varphi}^{\prime}-\tilde{\kappa}_{r+1} \cdot c+D_{r+1}^{*} \cdot u_{\varphi}^{\prime}+D_{r+1}^{* *} \cdot w_{\varphi}^{\prime}\right) ; \\
\lim _{\varphi \rightarrow \varphi_{* r-0}^{*}}(\tilde{\sigma} \cdot(c+u+w))^{\prime}{ }_{t}=\lim _{\varphi \rightarrow \varphi_{* r+0}^{*}}(\tilde{\sigma} \cdot(c+u+w))^{\prime}, r=\overline{1, p-1},
\end{array}\right. \\
& \left(\left.c\right|_{\eta=0, \psi=\widehat{\psi}}=\left.c\right|_{\eta=0, \psi=Q_{*}-\widehat{\psi}},\left.c_{\vec{n}}^{\prime}\right|_{\eta=0, \psi=\widehat{\psi}}=\left.c_{\vec{n}}^{\prime}\right|_{\eta=0, \psi=Q_{*}-\widehat{\psi}} ;\right. \\
& \left.c\right|_{\psi=0}=\left.c\right|_{\psi=Q_{*}},\left.\quad c_{\vec{n}}^{\prime}\right|_{\psi=0}=\left.c_{\vec{n}}^{\prime}\right|_{\psi=Q_{*}} ; \\
& \left.u\right|_{\eta=0, \psi=\widehat{\psi}}=\left.u\right|_{\eta=0, \psi=Q_{*}-\widehat{\psi}},\left.u_{\vec{n}}^{\prime}\right|_{\eta=0, \psi=\widehat{\psi}}=\left.u_{\vec{n}}^{\prime}\right|_{\eta=0, \psi=Q_{*}-\widehat{\psi}} ; \\
& \left\{\left.u\right|_{\psi=0}=\left.u\right|_{\psi=Q_{*}},\left.u_{\vec{n}}^{\prime}\right|_{\psi=0}=\left.u_{\vec{n}}^{\prime}\right|_{\psi=Q_{*}}\right. \\
& \left\{\left.w\right|_{\eta=0, \psi=\widehat{\psi}}=\left.w\right|_{\eta=0, \psi=Q_{*}-\widehat{\psi}},\left.w_{\vec{n}}^{\prime}\right|_{\eta=0, \psi=\widehat{\psi}}=\left.w_{\vec{n}}^{\prime}\right|_{\eta=0, \psi=Q_{*}-\widehat{\psi}} ;\right. \\
& \left.w\right|_{\psi=0}=\left.w\right|_{\psi=Q_{*}},\left.w_{\vec{n}}^{\prime}\right|_{\psi=0}=\left.w_{\vec{n}}^{\prime}\right|_{\psi=Q_{*}} ; \\
& \left.\tilde{T}\right|_{\eta=0, \psi=\widehat{\psi}}=\left.\tilde{T}\right|_{\eta=0, \psi=Q_{*}-\widehat{\psi}},\left.\tilde{T}_{\vec{n}}^{\prime}\right|_{\eta=0, \psi=\widehat{\psi}}=\left.\tilde{T}_{\vec{n}}^{\prime}\right|_{\eta=0, \psi=Q_{*}-\widehat{\psi}} ; \\
& \left.\tilde{T}\right|_{\psi=0}=\left.\tilde{T}\right|_{\psi=Q_{*}},\left.\quad \tilde{T}_{\vec{n}}^{\prime}\right|_{\psi=0}=\left.\tilde{T}_{\vec{n}}^{\prime}\right|_{\psi=Q_{*}}
\end{aligned}
$$

де $c=c(\varphi, \psi, \eta, t)=C(x(\varphi, \psi, \eta), y(\varphi, \psi, \eta), z(\varphi, \psi, \eta), t), \ldots, \widehat{\psi} \in\left[0, \frac{Q_{*}}{2}\right]$. 
Аналогічно [16] знайдено числово-асимптотичне наближення розв'язку задачі (14)-(18) з точністю $O\left(\varepsilon^{n+1}\right)$.

\section{2. РЕЗУЛЬТАТИ ЧИСЛОВИХ РОЗРАХУНКІВ}

У ході комп'ютерного експерименту під час моделювання процесу доочисти води від домішок у швидкому двошаровому фільтрі довжиною 1 м проведено дослідження зміни розподілу середніх величин концентрацій домішок у фільтраційній течії і адсорбованих з фільтраційної течії на поверхні адсорбенту завантаження відповідно при фізичній та хімічній адсорбції, середньої величини температури фільтраційної течії та середніх величин коефіцієнта фільтрації і активної пористості вздовж кусково-однорідного пористого завантаження фільтру. Форму модельного фільтру описано поверхнями $f_{*}(x, y, z)=x^{2}+y^{2}+z^{2}-4, f^{*}(x, y, z)=x^{2}+y^{2}+z^{2}-1$, $f^{* *}(x, y, z)=7,54863 \cdot x^{2}-y^{2}-z^{2}$ і вибрано положення поверхні розділу $E F F_{*} E_{*} f_{*}^{*}(x, y, z)=x^{2}+y^{2}+z^{2}-2,25$. Функції $f_{*}(x, y, z), f^{*}(x, y, z)$ та $f^{* *}(x, y, z)$ підібрані так, щоб забезпечити „монотонне звуження“ в напрямку від його входу до виходу та взаємну ортогональність граней уздовж ребер. При цьому кожен шар фільтру характеризується різними початковими значеннями коефіцієнтів фільтрації $\kappa_{1}^{0}=8,5$ м/добу, $\kappa_{2}^{0}=5,6$ м/добу, активної пористості $\tilde{\sigma}_{1}^{0}=0,41, \tilde{\sigma}_{2}^{0}=0,38$, дифузії домішок у фільтраційній течії $D_{1}=0,0003 \aleph^{2} /$ добу, $D_{2}=0,0002 \aleph^{2} /$ добу, дифузії домішок, адсорбованих з фільтраційного потоку на поверхні адсорбенту завантаження відповідно при фізичній та хімічній адсорбції $D_{1}^{*}=0,000005 \mathcal{м}^{2} /$ добу $D_{2}^{*}=$

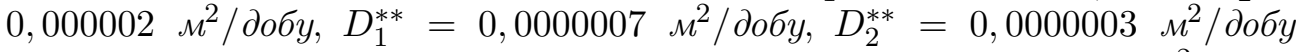
і температуропровідності фільтраційного потоку $D_{1}^{* * *}=0,0005 \mathrm{~s}^{2} /$ добу, $D_{2}^{* * *}=0,0003 \boldsymbol{м}^{2} /$ добу. У результаті побудовано розрахункову сіткову область $G_{\mathbf{z}}$ при $n=n_{1}+n_{2}=33, n_{1}=22, n_{2}=11, m=17, l=9$, $\varphi_{*}=\varphi_{0}=0, \varphi^{*}=\varphi_{33}=14,5$. Значення $\varphi^{*}$ вибиралося так, щоб середня швидкість фільтрації вздовж двошарового пористого середовища $v_{\text {сер }}(\varphi)$ становила $5 \mathrm{~m} /$ год. Для області $G_{\mathbf{z}}$ знайдено фільтраційну витрату $Q=84,1 \mathcal{M}^{3} /$ год, значення потенціалу на поверхні розділу $\varphi_{*}^{*}=\varphi_{22}=8,2$ та обчислено величини швидкостей фільтрації і функції $b_{i}(\varphi, \psi, \eta)(i=\overline{1,5})$.

На рис. 2 представлено розподіли середніх величин концентрацій домішок $c_{\text {сер }}(\varphi, t)$ у фільтраційній течії, концентрацій домішок $u_{\text {сер }}(\varphi, t)$ і $w_{\text {сер }}(\varphi, t)$, адсорбованих на поверхні адсорбенту завантаження відповідно при фізичній та хімічній адсорбції, температури $\tilde{T}_{\text {сер }}(\varphi, t)$, коефіцієнтів фільтрації $\tilde{\kappa}_{\text {сер }}(\varphi, t)$ і активної пористості $\tilde{\sigma}_{\text {сер }}(\varphi, t)$ вздовж висоти модельного фільтру у момент часу $t_{3}=23$ год при $c_{\kappa \mathrm{p}}=0,00005$ г/л, $c_{*}=$ $0,005 \imath / \Omega, c_{0}^{0}=0, u_{0}^{0}=0, w_{0}^{0}=0, \tilde{T}_{0}^{0}=20^{\circ} C, \tilde{\alpha}_{1}=0,3-0,01 \cdot \tilde{v} c^{-1}, \tilde{\alpha}_{2}=$ $0,38-0,01 \cdot \tilde{v} c^{-1}, \tilde{\beta}_{s}=0,00001 \cdot \tilde{\alpha}_{s} c^{-1}(s=1,2), \tilde{\alpha}_{1}^{*}=0,25-0,002 \cdot \tilde{v} c^{-1}$, $\tilde{\alpha}_{2}^{*}=0,35-0,001 \cdot \tilde{v} c^{-1}, \tilde{\mu}_{1}=0,000025 \Omega /(2 \cdot c), \tilde{\mu}_{2}=0,000023 \Omega /(2 \cdot c)$, $\tilde{\mu}_{1}^{*}=0,000025 \Omega /(z \cdot c), \tilde{\mu}_{2}=0,000023 \Omega /(z \cdot c), \tilde{\lambda}_{1}=0,000031 \Omega /(z \cdot c)$, $\tilde{\lambda}_{2}=0,000028 \Omega /(2 \cdot c), \tilde{\lambda}_{1}^{*}=0,000021 \Omega /\left((2 \cdot c), \tilde{\lambda}_{2}^{*}=0,000028 \Omega /(a \cdot c)\right.$, $\tilde{\gamma}_{1}=1200{ }^{\circ} C \cdot \Omega / 2, \tilde{\gamma}_{2}=1500{ }^{\circ} C \cdot \Omega / \imath, \tilde{\gamma}_{1}^{*}=60000{ }^{\circ} C \cdot \Omega / \imath, \tilde{\gamma}_{2}^{*}=80000{ }^{\circ} C \cdot \Omega / \imath$, $\tilde{\theta}=0,1$. 
Результати числового експерименту по розрахунку часу захисної дії фільтра при різних значеннях гранично допустимої концентрації домішок без відведення та з урахуванням відведення частини тепла на поверхні розділу наведені у табл. 1. 3 отриманих результатів можна зробити висновок, що відведення частини тепла на поверхні розділу сприяє збільшенню часу захисної дії фільтра, так при $c_{\kappa р}=0,05$ мг/ $\Omega$ час захисної дії фільтру збільшився на 8 годз 15 год до 23 год, а при $c_{\kappa р}=0,1$ мг/л- на 16 годз 32 год до 48 год.

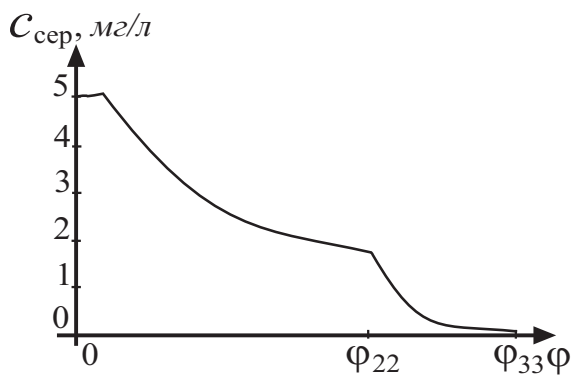

a)

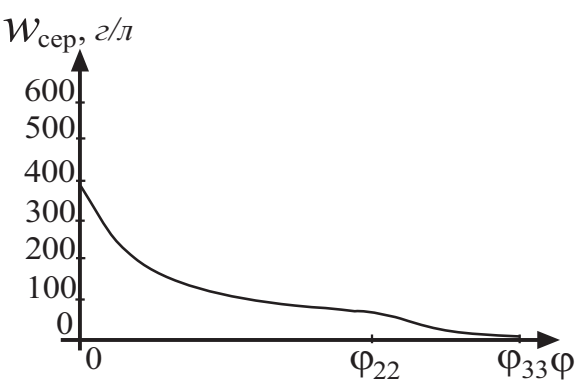

б)

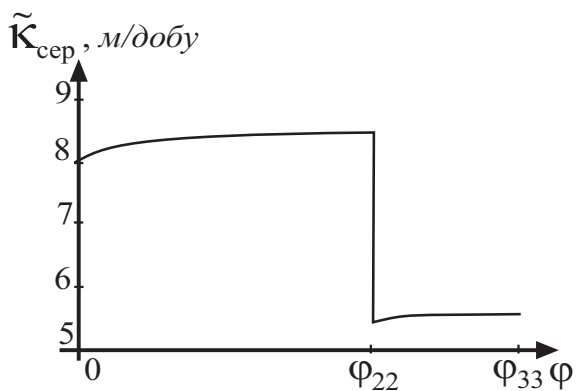

д)

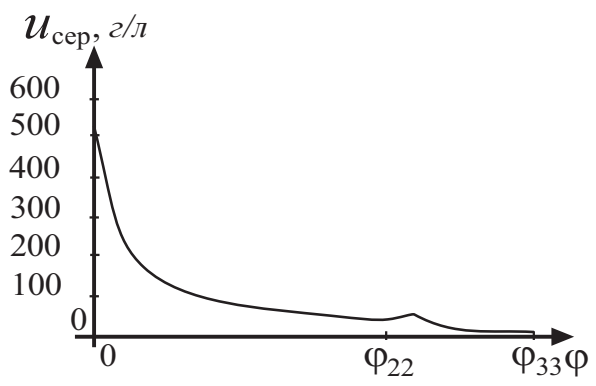

б)

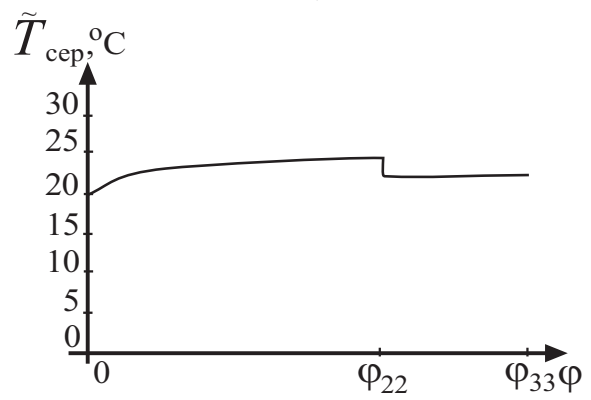

г)

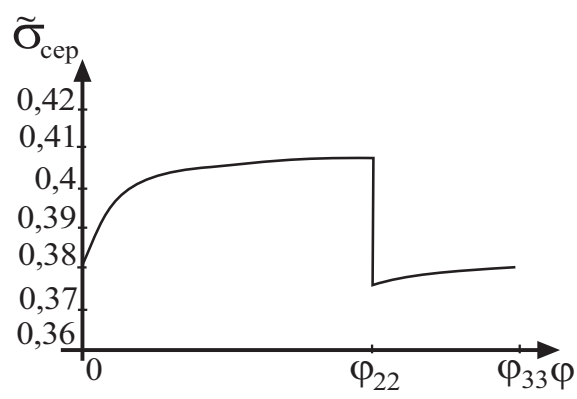

e)

Рис 2. Розподіл середніх величин концентрацій домішок у фільтраційній течії (а), осівших на поверхні адсорбенту завантаження за рахунок фізичної (б) та хімічної адсорбції (в), температури фільтраційної течії (г), коефіцієнтів фільтрації (д) та активної пористості (е) вздовж висоти фільтру у момент часу $t_{3}=23$ год 
ТАБлиця 1. Розрахований час захисної дії модельного фільтра

\begin{tabular}{|c|c|c|c|}
\hline № & $c_{\kappa \mathrm{p}}$, мг/ $/$ & $t_{3}$ без відведення, год & $t_{3}$ з відведенням, год \\
\hline 1 & 0,05 & 15 & 23 \\
\hline 2 & 0,06 & 18 & 28 \\
\hline 3 & 0,07 & 22 & 33 \\
\hline 4 & 0,08 & 25 & 37 \\
\hline 5 & 0,09 & 28 & 42 \\
\hline 6 & 0,1 & 32 & 48 \\
\hline
\end{tabular}

\section{ВисновКИ}

Сформовано математичну модель для комп'ютерного прогнозування процесу адсорбційної доочистки води від домішок у швидких багатошарових фільтрах конусоподібної форми з кусково-однорідними пористими завантаженнями при додержанні сталої швидкості фільтрування з урахуванням зворотного впливу характеристик процесу (концентрацій домішок у фільтраційній течії та адсорбованих на поверхні адсорбенту завантаження за рахунок фізичної і хімічної адсорбції, температури) на характеристики завантаження (коефіцієнти фільтрації й активної пористості адсорбентів у кожному шарі, коефіцієнти, які характеризують швидкості масообміну при фізичній та хімічній адсорбції домішок і фізичній десорбції домішок) та 3 урахуванням відведення частини тепла з поверхнонь розділу шарів. Запропонована модель аналогічно $[18,19]$ забезпечує можливість вивчення зміни концентрацій домішок у фільтраційній течії та адсорбованих на поверхні адсорбенту завантаження відповідно при фізичній та хімічній адсорбції, температури, характеристик кусково-однорідних пористих завантажень вздовж висоти фільтру та на їх основі прогнозування більш оптимальних варіантів використання адсорбентів і збільшення тривалості роботи фільтрів. Результати числових експериментів (табл. 1) свідчать про збільшення часу захисної дії фільтра приблизно у 1,5 рази.

\section{ЛiтератУра}

1. Двадненко М. В., Привалова Н. М., Кудаева И. Ю., Степура А. Г. Адсорбционная очистка сточных вод. Современные наукоемкие технологии. 2010. № 10. C. $214-215$.

2. Edzwald J. Water Quality \& Treatment. A Handbook on Drinking Water. McGraw-Hill Professional, 2010. 1996 p.

3. Hendricks D. W. Fundamentals of water treatment unit processes: physical, chemical, and biological. Boca Raton : CRC Press, 2011. - 883 p.

4. Сакалова Г. В., Василінич Т. М. Дослідження ефективності очищення стічних вод від іонів важких металів з використанням природних адсорбентів: монографія. Вінниця: ТОВ „Твори“, 2019. 92 с.

5. Невзорова А. Б. Основы автоматизации систем водоснабжения и водоотведения: пособие. Гомель: УО „БелГУТ“, 2005. 115 с.

6. Макаревич Н. А., Богданович Н. И. Теоретические основы адсорбции: учебное пособие. Архангельск: САФУ, 2015. 362 с. 
7. Зеленцов В. И., Дацко Т. Я., Дворникова Е. Е. Влияние температуры на равновесие и кинетику адсорбции фторид-ионов термообработанными оксигидратами Al. Электронная обработка материалов. 2008. № 3. С. 50-58.

8. Лаптев А. Г., Сергеева Е. С. Физическое и математическое моделирование сорционных свойств торфа для очистки воды от нефтепродуктов. Вода: химия и екология. 2009. № 3. С. 14-19.

9. Sperlich A., Schimelpfenning S., Baumgarten B. et al. Predicting anion breakthrough in granular ferric hydroxide (GFH) adsorption filters. Water Res. 2008. 42. P. 2073-2082.

10. Бомба А. Я., Сафоник А. П. Моделювання нелінійно-збурених процесів очищення рідин від багатокомпонентних забруднень: монографія. Рівне: НУВГП, 2017. 296 c.

11. Поляков В. Л. Моделирование процесса водоочистки в загрузке адсорбера. Доповіді Національної академї наук України. 2012. № 5. С. 63-71.

12. Kalteh A. M., Hjorth P. and Berndtsson R. Review of the self-organizing map (SOM) approach in water resources: analysis, modelling and application. Environmental Modelling and Software. 2008. Vol. 23. № 7. P. 835-845.

13. Maier H. R., Dandy G. C. Neural networks for the prediction and forecasting of water resources variables: a review of modelling issues and applications. Environmental Modelling and Software. 2000. Vol. 15. № 1. P. 101-124.

14. Heikkinen M., Poutiainen H., Liukkonen M., Heikkinen T. and Hiltunen Y. Selforganizing maps in the analysis of an industrial wastewater treatment process. Mathematics and Computers in Simulation. 2011. Vol. 82. № 3. P. 450-459.

15. Safonyk A. P. Modelling the filtration processes of liquids from multicomponent contamination in the conditions of authentication of mass transfer coefficient. International Journal of Mathematical Models and Methods in Applied Sciences. 2015. Vol. 9. P. 189-192.

16. Климюк Ю. Є., Бомба А. Я. Комп'ютерне прогнозування адсорбційної доочистки води від домішок у швидких багатошарових фільтрах конусоподібної форми. Математичне та комп'ютерне моделювання. Серія: Технічні науки. Вип. 20. Кам'янець-Подільськ : Кам'янець-Подільський національний університет імені Івана Огієнка, 2019. С. 51-68.

17. Klimjuk Ju. Je. Modelling of spatial filtration fields for one class of rapid multilayer filters of cone-shaped form with piecewise-homogeneous porous loads. Proceedings of III International scientific conference „Science: new goals“. London: SI Universum, 2017. P. 4-10.

18. Бомба А. Я., Климюк Ю. Є. Прогнозування зміни характеристик кусковооднорідних пористих завантажень швидких багатошарових фільтрів. Вісник НТУ „ХПІ“. Серія: Математичне моделювання в техніці та технологіях. Харків : НТУ „ХПІ“, 2018. № 27 (1303). С. 8-15.

19. Бомба А. Я., Климюк Ю. Є. Прогнозування оптимального використання фільтруючих матеріалів у швидких багатошарових фільтрах із кусковооднорідними пористими завантаженнями. Журнал обчислювальной та прикладної математики: наук. журн. Київ. нац. ун-т ім. Тараса Шевченка. Вип. 1 (124). Київ: ТВіMC, 2017. С. 3-14. 


\title{
КОМПЬЮТЕРНОЕ ПРОГНОЗИРОВАНИЕ ПОВЫШЕНИЯ ЭФФЕКТИВНОСТИ РАБОТЫ БЫСТРЫХ МНОГОСЛОЙНЫХ ФИЛЬТРОВ ДЛЯ АДСОРБЦИОННОЙ ДООЧИСТКИ ВОДЫ
}

\author{
А. Я. БОмБА, Ю. Е. КлимЮК
}

Кафедра компьютерных наук и прикладной математики, Национальный университет водного хозяйства и природопользования, Ровно, Украина,

E-mail: \{abomba, klimyuk\}@ukr.net

АннотАция. Предложена математическая модель адсорбционной доочистки воды от примесей в быстрых многослойных фильтрах конусообразной формы с кусочно-однородными пористыми загрузками при соблюдении постоянной скорости фильтрования с отводом части тепла с поверхности раздела слоев. Модель обеспечивает возможность проведения компьютерных экспериментов по изучению изменения концентраций примесей в фильтрационном течении и адсорбированных на поверхности адсорбента загрузки при физической и химической адсорбции, температуры, характеристик кусочно-однородных пористых загрузок вдоль высоты фильтра и на их основе прогнозирования оптимальных вариантов использования адсорбентов и увеличение продолжительности работы фильтров.

КЛЮчЕВЫЕ словА: математическая модель, доочистка воды, примесь, адсорбция, быстрый многослойный фильтр. 